\title{
Neutral Flow Evolution in a Six-Kilowatt Hall Thruster
}

\author{
Wensheng Huang* and Alec D. Gallimore \pm \\ University of Michigan, Ann Arbor, Michigan 48109 \\ and \\ Richard R. Hofer声 \\ Jet Propulsion Laboratory, California Institute of Technology, Pasadena, California 91109 \\ DOI: $10.2514 / 1 . B 34048$
}

\begin{abstract}
Laser-induced fluorescence velocimetry measurements obtained from the interior of a 6-kW Hall thruster using the Xe I $6 s[3 / 2]_{2}^{0} \rightarrow 6 p[3 / 2]_{2}$ transition at $823.4 \mathrm{~nm}$ (vacuum) and the Xe I $6 s^{\prime}[1 / 2]_{1}^{0} \rightarrow 6 p^{\prime}[3 / 2]_{2}$ transition at $834.9 \mathrm{~nm}$ (vacuum) are presented. The thruster is operated under seven conditions, with discharge voltages ranging from 150 to $600 \mathrm{~V}$ and anode mass flow rates ranging from 10 to $30 \mathrm{mg} / \mathrm{s}$. Velocimetry results along the channel centerline show that the neutral propellant leaves the anode at a bulk axial velocity of $\sim 100 \mathrm{~m} / \mathrm{s}$ and accelerates to $300-400 \mathrm{~m} / \mathrm{s}$ by the exit plane. The temperature of the neutrals starts out at 1000-1600 K near the anode but cools down to 500-800 K near the exit plane. The anode mass flow rate appears to have a bigger influence on the bulk velocity and temperature of the neutrals than the discharge voltage. Radial sweeps across the channel exit plane revealed the presence of near-wall boundary layers approximately a few millimeters thick. The experimental results are compared with MONACO and HPHall-2 simulation results. The comparison suggests that the neutral-flow acceleration is due to a combination of wall thermalization, hydrodynamic, and ionization effects.
\end{abstract}

\section{Introduction}

I N TYPICAL Hall thruster studies, information about the neutral flow is obtained using fluid and particle simulations. However, there is a gap in knowledge regarding how the plasma affects the behavior of neutrals. Several studies in the past have shown that neutral-flow mechanics can play a very important role in controlling the location and properties of the plasma in a Hall thruster [1]. Because of a lack of neutral-flow data, it is difficult to judge the fidelity with which neutral-flow behavior is captured in simulations. Laser-induced fluorescence (LIF) presents a unique technique for bridging the knowledge gap, in that it is nonintrusive, species specific, and capable of extracting information about the neutrals in the Hall thruster discharge channel. There have been several previous neutral-flow LIF studies on magnetic-layer thrusters [2,3] including internal flow measurements obtained through a slot in the channel [4]. This paper will present neutral-flow data from the interior of a 6-kW magnetic-layer Hall thruster operating on xenon. The data are obtained without the use of a slot and are truly nonintrusive. This paper will begin by describing the theories associated with LIF velocimetry, the experimental setup, data reduction, and empirical results. It will then describe several MONACO and HPHall-2 simulations for comparison against the empirical data. Lastly, the paper will discuss the physical trends and mechanisms that best explain the results.

\section{Theories}

This section describes several basic principles for those not familiar with LIF velocimetry. For the remainder of this paper, all

Received 15 June 2010; revision received 20 December 2010; accepted for publication 30 December 2010. Copyright (C) 2011 by the American Institute of Aeronautics and Astronautics, Inc. All rights reserved. Copies of this paper may be made for personal or internal use, on condition that the copier pay the $\$ 10.00$ per-copy fee to the Copyright Clearance Center, Inc., 222 Rosewood Drive, Danvers, MA 01923; include the code 0748-4658/11 and $\$ 10.00$ in correspondence with the CCC.

${ }^{*} \mathrm{Ph}$. D. Candidate, Plasmadynamics and Electric Propulsion Laboratory, Aerospace Engineering; davhuang@umich.edu. Student Member AIAA.

$\dagger$ Arthur F. Thurnau Professor of Aerospace Engineering, Lab Director Plasmadynamics and Electric Propulsion Laboratory; Alec.Gallimore@ umich.edu. Fellow AIAA.

${ }^{\ddagger}$ Senior Engineer, Electric Propulsion Group; richard.r.hofer@jpl.nasa. gov. Senior Member AIAA. transition wavelengths are given in vacuum values and all intensities given in arbitrary units (a.u.), unless otherwise specified.

LIF velocimetry operates on the principle that a particle (atom or molecule) absorbs a laser photon at a shifted frequency when moving due to the Doppler effect. The particle has a chance of spontaneously deexciting by emitting a photon. The emission, called fluorescence, is isotropic. For a particle traveling at nonrelativistic speed, the shift in absorption frequency is proportional to the component of particle velocity in the direction that the photon travels. By varying the frequency of the injected photons and comparing the intensity of the collected fluorescence, we can obtain the particle velocity distribution function (VDF) along the injected photon wave vector.

In the first experiment, light is injected at $823.4 \mathrm{~nm}$ to excite the $6 s[3 / 2]_{2}^{0} \rightarrow 6 p[3 / 2]_{2}$ transition, and the fluorescence is collected resonantly at the same wavelength. In the second experiment, light is injected at $834.9 \mathrm{~nm}$ to excite the $6 s^{\prime}[1 / 2]_{1}^{0} \rightarrow 6 p^{\prime}[3 / 2]_{2}$ transition, and the fluorescence is collected nonresonantly at $473.5 \mathrm{~nm}$. Figure 1 shows the associated transition diagram. Each of these two transitions has its own advantages and disadvantages. The lower state of the Xe I $823.4 \mathrm{~nm}$ transition is metastable, and the signal is exceptionally strong. This line shows effect of saturation under typical experimental conditions. Physically, saturation occurs when the upper state population is sizable compared with the lower state population so that the rate of laser-induced excitation no longer scales linearly with the laser intensity. A more rigorous description of this phenomenon can be found in [5]. An easily saturated line can be useful for measuring relative density, because the fluorescence signal becomes less sensitive to input laser power. However, the saturation effect leads to broadening that distorts the line shape so that only the most probable velocity can be extracted. Furthermore, since the $823.4 \mathrm{~nm}$ LIF scheme is resonant, the signal can be distorted by surface scatter, making it impossible to collect data too close to the walls and the anode.

The Xe I $834.9 \mathrm{~nm}$ LIF scheme has the advantage of being nonresonant and can be used to take measurements near the thruster walls and the anode. However, the associated lower state is not metastable, and the signal tends to be weaker than the Xe I $823.4 \mathrm{~nm}$ scheme. For the Xe I $834.9 \mathrm{~nm}$ LIF scheme, we typically obtain unsaturated line shapes with the equipment described herein so VDFs can be extracted. Note that we are directly measuring the VDF of the lower states of the LIF transitions, which is assumed to have the same VDF as the entire neutral population. 


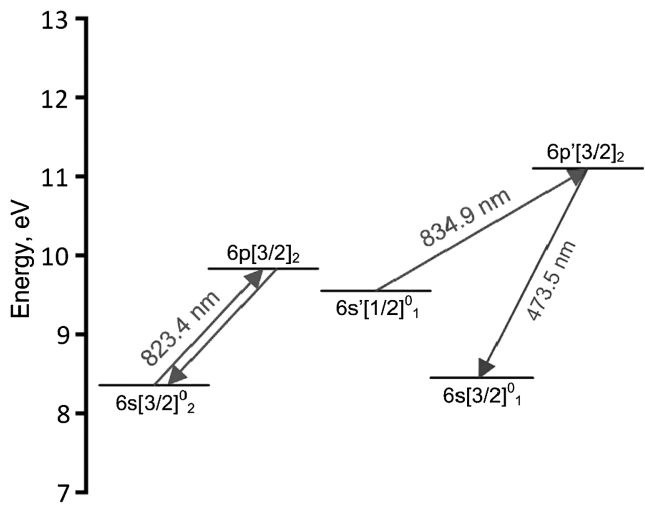

Fig. 1 Transition diagram for the two experiments. Wavelengths in vacuum.

The full-width-at-half-maximum (FWHM) value of the average neutral VDF in a Hall thruster typically varies from 400 to $800 \mathrm{~m} / \mathrm{s}$, which for a near-infrared transition corresponds to about 500 to $1000 \mathrm{MHz}$ in frequency space. Unfortunately, the xenon hyperfine structures for the two transitions used in this study are up to several gigahertz wide. These structures must be accounted for in order to extract the VDFs. The theory of hyperfine structures are described in [6,7]. Associated hyperfine structure constants are published in [810]. Further details regarding how the hyperfine structure theory applies to the $6 s[3 / 2]_{2}^{0} \rightarrow 6 p[3 / 2]_{2}$ and $6 s^{\prime}[1 / 2]_{1}^{0} \rightarrow 6 p^{\prime}[3 / 2]_{2}$ transitions can be found in [11].

\section{Experimental Setup}

This section describes various components of the experimental setup in detail.

\section{A. Thruster}

The 6-kW annular magnetic-layer Hall thruster used for this investigation nominally operates at $300 \mathrm{~V}$ discharge voltage and $20 \mathrm{mg} / \mathrm{s}$ anode mass flow rate [1]. Table 1 lists the operating conditions tested during this experiment. Note that the discharge voltage and anode mass flow rate are the main varying parameters between conditions. For the rest of this paper, operating conditions are labeled as $Y Y Y-V$ and $Z Z \mathrm{mg} / \mathrm{s}$, where $Y Y Y$ is the discharge voltage and $Z Z$ is the anode mass flow rate. Cathode mass flow rate is fixed to $7 \%$ of the anode mass flow rate for all operating conditions. Magnetic field lines are roughly symmetric about the thruster channel centerline for all conditions, and magnetic field strength is set to maximize thruster efficiency. These settings were previously found through the use of an inverted pendulum thrust stand. Research-grade xenon propellant (99.999\% pure) is supplied to the thruster by commercially available flow meters and controllers, having an accuracy of $\pm 1 \%$. Calibration of the flow system is done by the constant volume method, taking into account the effects of xenon compressibility.

\section{B. Air-Side Optics}

Figure 2 shows the airside laser and optics setup for the LIF experiment. The laser system used is a TOPTICA TA-100/830 tapered-amplifier diode laser. This system has a nominal linewidth of

Table 1 List of operating conditions

\begin{tabular}{ccc}
\hline \hline Discharge voltage, $\mathrm{V}$ & Anode mass flow rate, $\mathrm{mg} / \mathrm{s}$ & Discharge current, A \\
\hline 150 & 10 & 9.08 \\
150 & 20 & 21.55 \\
150 & 30 & 35.35 \\
300 & 10 & 8.94 \\
300 & 20 & 20.11 \\
300 & 30 & 33.25 \\
600 & 10 & 9.36 \\
\hline
\end{tabular}

${ }^{\mathrm{a}}$ Uncertainty in discharge current is $\pm 0.5 \%$.

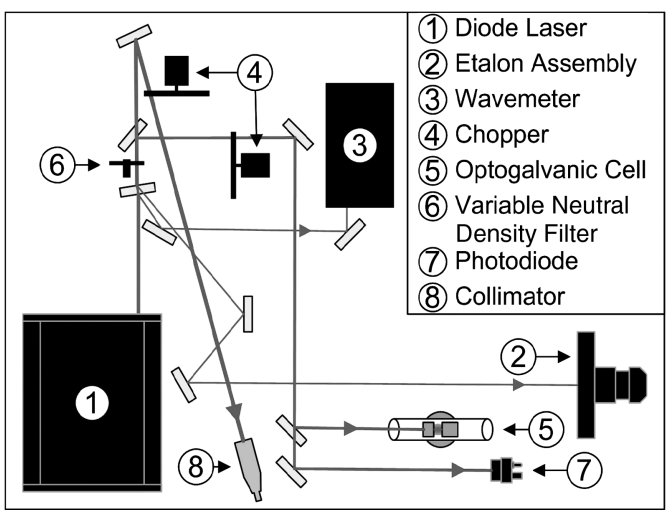

Fig. 2 Air-side laser injection setup.

$\sim 10 \mathrm{MHz}$ and a mode-hop-free range of $\sim 12 \mathrm{GHz}$. Output power is $\sim 250 \mathrm{~mW}$ at the wavelengths used in this study. The main laser beam is mechanically chopped at $\sim 1.8 \mathrm{kHz}$ via an SR540 chopper, and it is sent into a fiber collimator. The fiber delivers the light through a feedthrough into the vacuum chamber.

For reference information, a Burleigh SA-91 etalon assembly ( $2 \mathrm{GHz}$ free spectral range, finesse greater than 300), a Burleigh WA1000 wavemeter (accurate to $1 \mathrm{pm}$ ), and a Hamamatsu L2783-42 XeNe-Mo optogalvanic cell are used. The optogalvanic cell is made of two cylindrical Mo tube electrodes centered in a glass cylinder filled with approximately 3 torr of xenon and 4 torr of neon. The cell is operated at $250 \mathrm{~V}$. The voltage drop across the cell's ballast resistor is connected to an SR-810 lock-in amplifier through a resistorcapacitor high-pass filter.

Additionally, a Thorlabs DET-110 photodiode is used to monitor laser power drift. This reading is fed into another SR-810 lock-in amplifier for signal collection. For unsaturated LIF traces, this laser power reading is used to remove the effects of laser power drift in postprocessing. When doing a saturation study, an additional variable neutral density filter is added so that the laser power being injected into the chamber can be controlled.

Lastly, on the airside, an optical fiber brought the fluorescence signal from the chamber to a SPEX-500M monochromator, the output of which is fed to a Hamamatsu R928 photomultiplier. The amplified signal is sent to a third SR-810 lock-in amplifier. The monochromator slit width is $1 \mathrm{~mm}$, corresponding to an optical bandwidth of $1 \mathrm{~nm}$.

\section{Facility and Vacuum-Side Optics}

Experiments are performed in the Large Vacuum Test Facility (LVTF) of the Plasmadynamics and Electric Propulsion Laboratory at the University of Michigan. The LVTF is a $\varphi 6 \times 9 \mathrm{~m}$ stainlesssteel-clad cylindrical steel chamber. The thruster plume is allowed to expand unimpeded until termination at a carbon beam dump $\sim 4 \mathrm{~m}$ downstream. Pumping is provided by seven cryopumps with a nominal xenon pumping speed of $245,000 \mathrm{l} / \mathrm{s}$. Facility pressure is monitored by two hot-cathode ionization gauges. The base pressure is $\sim 2 \times 10^{-7}$ Torr. The pressure during thruster operation at a $30 \mathrm{mg} / \mathrm{s}$ anode mass flow rate is $\sim 2 \times 10^{-5}$ Torr, corrected for xenon [12].

During the experiments, the 6-kW Hall thruster is mounted on two cross-mounted stepper-motor translation stages. The stages provide $1 \mathrm{~m}$ of axial travel, $2 \mathrm{~m}$ of radial travel, and have a positioning accuracy of $\pm 0.1 \mathrm{~mm}$. Laser injection and LIF collection optics are fixed to the floor of the chamber. Two different types of spatial sweeps are carried out. The axial sweeps along the channel centerline revealed the evolution of the neutral-flow bulk velocity and temperature from the anode to the channel exit plane. The radial sweeps across the channel exit plane of the thruster revealed the difference in properties between the neutral flow near the walls and at the channel center.

Figure 3 shows a diagram of the vacuum-side experimental setup. The injection optics send the laser beam axially into the thruster. This 


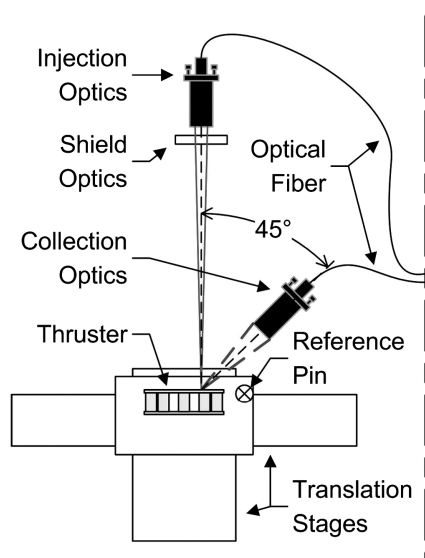

a)

Fig. 3 Vacuum-side experimental setups: a) Xe I $823.4 \mathrm{~nm}$ experiment and b) depiction of how the collection optics were repositioned for the Xe II 834.9 nm experiment.

beam is focused down to $1 \mathrm{~mm}$ in beam waist diameter via an antireflection-coated achromatic lens. A polarizer (not shown in diagram) is placed between the optical fiber output and the lens to provide a horizontal polarization with respect to the chamber floor to minimize magnetic hyperfine distortions. It was previously shown in [13] that, for a magnetic field strength of a few $100 \mathrm{G}$, the magnetic hyperfine effect is negligible when the polarization of the laser is parallel to the local magnetic field. The injection beam focusing cone has a half-angle of $\sim 0.5^{\circ}$, and the injection axis is aligned to within $0.1^{\circ}$ of the thruster firing axis, so cosine losses are negligible. The interrogation zone is at the nine o'clock position when viewing the thruster face on. To prevent excess thermal drift during thruster operation, an optical shield is installed to allow the laser to pass through while blocking the incoming xenon particles.

The collection optics are built from a matching pair of antireflection-coated achromatic lenses. Prior thermocouple measurements showed that thermal shielding is not necessary for the collection optics. Nevertheless, some thermal drift takes place during the test, so a reference pin is installed to the left of the thruster viewed face on. The exact position of this pin relative to the thruster is known, and the reflected laser signal from this pin is used to compensate for thermal drift.

For the Xe I $823.4 \mathrm{~nm}$ experiment, the collection optics are set at a $45 \pm 1^{\circ}$ angle with respect to the thruster firing axis (Fig. 3a) to allow the interrogation of the interior of the thruster channel. These optics are located at a distance of $\sim 300 \mathrm{~mm}$ away from the interrogation zone and have an effective light collection diameter of $\sim 50 \mathrm{~mm}$. The resulting intersection of the injection and collection optics forms a diagonally sliced cylinder $\sim 1 \mathrm{~mm}$ in diameter in the radial direction and $\sim 1.5 \mathrm{~mm}$ long in the axial direction. For the Xe I $834.9 \mathrm{~nm}$ experiment, the collection optics are set at a different angle to allow measurement all the way up to the anode. They are set at a $66 \pm 1^{\circ}$ with respect to the firing axis and at $20 \pm 1^{\circ}$ with respect to the vertical when viewing the thruster face on to maximize the depth into the channel from where we can collect data (Fig. 3b). The interrogation zone for this second setup is $\sim 1.5 \mathrm{~mm}$ in diameter and $\sim 1.5 \mathrm{~mm}$ long. The size of the interrogation zone sets the spatial resolution of the LIF measurements.

\section{Experimental Parameters and Saturation Studies}

During the experiments, all scans were taken with a lock-in amplifier time constant of $100 \mathrm{~ms}$ and a total scan duration of $160 \mathrm{~s}$. Amplifier settings and photomultiplier tube bias voltage are fixed throughout for each experiment to ensure data are taken under comparable conditions. Laser beam power delivered to the interrogation volume was measured via a thermopile to be $20 \pm 1 \mathrm{~mW}$, giving an average beam intensity of $\sim 2.5 \mathrm{~W} / \mathrm{cm}^{2}$.

Figures $4 \mathrm{a}$ and $4 \mathrm{~b}$ show the results of the 823.4 and $834.9 \mathrm{~nm} \mathrm{LIF}$ saturation studies, respectively. These results show that the sample is lightly saturated by the laser beam (slight nonlinearity in the curve fit) for the $823.4 \mathrm{~nm}$ LIF experiment and is not saturated for the $834.9 \mathrm{~nm}$ LIF experiment. Although the $823.4 \mathrm{~nm}$ line is only lightly saturated, there is still enough saturation effect that the VDF cannot be extracted without distortion. Strictly speaking, we are not performing saturation spectroscopy in the sense that the $823.4 \mathrm{~nm}$ transition is not well saturated; care had to be taken to keep the laser power to within $5 \%$ between scans. But, as we will discuss in Sec. V.A, other sources of uncertainty dominate over the influence of laser power on the relative density measurements.

\section{Simulation Setup}

\section{A. HPHall-2}

HPHall-2 is a hybrid/particle-in-cell (PIC) code designed specifically to simulate Hall thruster plasma. The plasma can be turned off to simulate the behavior of the neutral flow only. HPHall was originally developed by Fife [14]. The code was later updated to version 2.0 by Parra et al. [15]. The version used in this study is the Jet Propulsion Laboratory-modified version of HPHall-2 [16]. Only the 300-10, 300-20, and 300-30 operating conditions were simulated. The results with the plasma turned on are from Reid's dissertation [1]. The results with the plasma turned off are specially simulated for this paper. Anode and wall temperatures are set to constants based on prior knowledge from Reid and on the results of a calibrated infrared imaging study by Mazouffre et al. [17]. Specifically, the values of the wall temperature are 780,940 , and $1060 \mathrm{~K}$, and the values of the anode temperature are 680,840 , and $960 \mathrm{~K}$ for the 300-10, 300-20, and 300-30 operating conditions, respectively. We use temperatures from plasma-on conditions in order to study the effect of thermalization separate from other plasma effects. Details on how
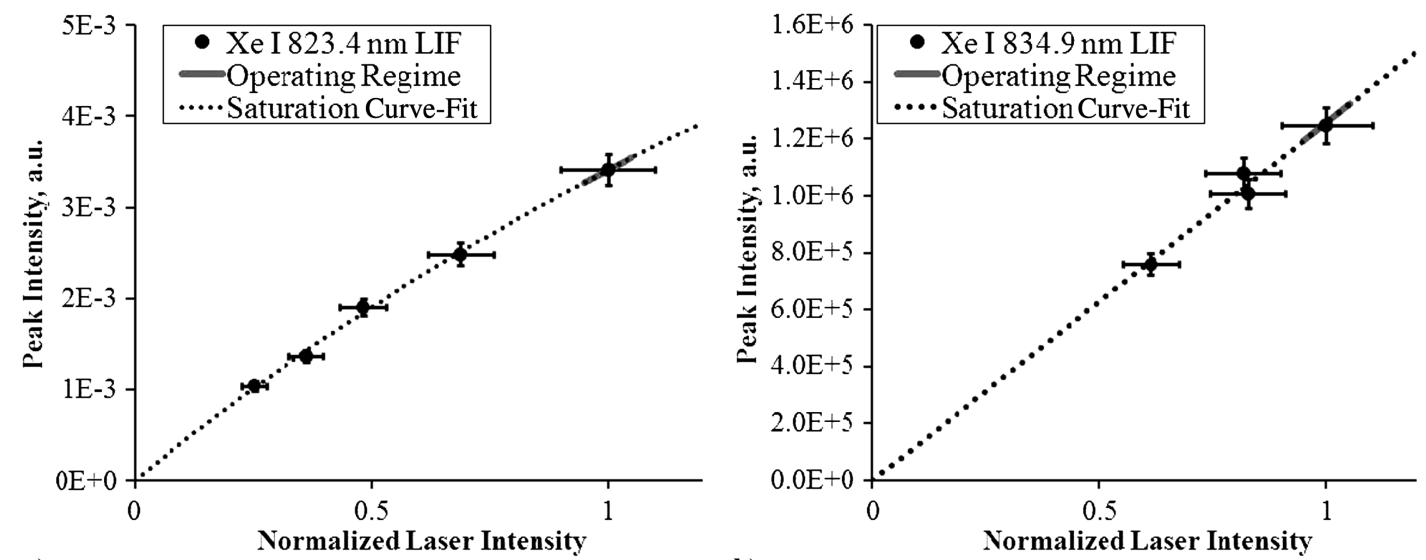

b)

Fig. 4 Saturation study results. 
HPHall-2 handles neutral-flow boundary conditions and dynamics are described in [18].

\section{B. MONACO}

MONACO version 3.0 is a PIC code designed to simulate general rarefied flow. It was originally developed by Dietrich and Boyd [19]. MONACO is used to provide comparison simulation data for thruster operation with the plasma turned off and to study the sensitivity of the neutral-flow profile to changes in anode and wall temperatures. The same geometry and boundary conditions are used for MONACO simulations as in the HPHall-2 simulations, although the domain of the HPHall-2 simulations isotropically extend about two channel widths past the channel exit plane, while the domain of the MONACO simulations stops at the channel exit plane.

\section{Data Analysis and Results}

\section{A. Data Reduction}

All axial positions are normalized by the discharge channel length, and negative axial positions are inside the channel. The exit plane is located at 0 , and the anode is located at -1 . Radial positions are normalized by the discharge channel width. The inner wall is located at zero, while the outer wall is located at one. The signal-to-noise ratio (SNR) of an LIF trace is defined as the peak intensity divided by the root-mean-square value of the noise fluctuations. The SNR of a trace is considered too low if it is less than 10 , because at this point, the noise contributes significantly to the uncertainty of the velocity measurements.

For the Xe I $823.4 \mathrm{~nm}$ experiment, parabolas are fitted to the main peak of the LIF and stationary reference line shapes, then the difference in spectral frequencies is converted to equivalent velocity. Only data points with intensities greater than $70 \%$ of the peak intensity are used in the curve fit to guarantee that the fit is only applied to the main peaks. This method yields the most probable velocity of the neutrals. The uncertainty associated with the measured most probable velocity is $\pm 50 \mathrm{~m} / \mathrm{s}$ based on the frequency resolution of the LIF system. Relative density is assumed to be proportional to the maximum intensity of the main peak based on the same curve fit. Care is taken to make sure all data points for each set of relative intensity data are taken under the same test conditions. The main peak is ideal for relative density measurements because it is the most saturated of the four prominent peaks for this transition. The ratio between the density of the $6 s[3 / 2]_{2}^{0}$ metastable state to that of all neutrals is a function of plasma temperature, density, and (by extension) collisionality. Usually, a collisional-radiative model is needed to correlate relative change in density of the excited state to the overall change in density of the neutrals. However, for a Hall thruster with a well-confined plasma, like the one found in the test article, the neutrals ionize over a very short distance (characteristic neutral-density-gradient length scale shorter than the collisional mean free path). Under this condition, the thermodynamic state of the neutral xenon population is roughly the same before and after the ionization zone. To a first-order approximation, the proportionality constant between the LIF signal strength and the overall neutral xenon density stays roughly constant throughout the ionization zone. We emphasize that the relative density measurements are very rough estimates, since the ratio between the density of the $6 s[3 / 2]_{2}^{0}$ metastable state and the rest of the neutral population does change between axial data locations. This is the greatest source of uncertainty in our relative density measurement, and we estimate it to be roughly $\pm 20 \%$. We note here that the relative density measurements are more qualitative than quantitative due to the large uncertainty, but that these measurements are nevertheless a good indicator of the ionization location, because the fluorescence intensity changes by one to two orders of magnitude over a few axial data locations. Simple geometric calculations show that the collection optics view cone intersects the outer wall of the discharge channel for $Z<-0.3$. Data taken upstream of $Z=-0.3$ are therefore not useful for relative uncertainty measurement.
For the Xe I $834.9 \mathrm{~nm}$ experiment, it is found that the shift in frequency due to the average movement of the particles is small compared with the frequency width associated with various broadening factors. The best way to obtain bulk velocity (mean velocity) in this case is to perform direct averaging of the LIF and stationary reference line shapes. This approach works for the second experiment but not the first, because the LIF line shapes are unsaturated for the second experiment. Deconvolution of hyperfine structures from the raw LIF line shapes is carried out using a Fourier transform method with an inverse Gaussian filter. This method is described in detail by Smith [20]. Temperatures are obtained by first calculating the FWHM velocity of the VDF, then finding a Maxwellian distribution for which the temperature gives rise to the same FWHM velocity. This approach is useful because the VDFs are slightly nonMaxwellian, with the majority of the distortion happening on the two wings of the distribution. The distortion in the wings can lead to erroneous temperatures if a simple Maxwellian fit is performed. For the most part, the data are taken from the anode all the way to the exit plane along the centerline. However, the signal tends to fall off quickly as the interrogation zone nears the exit plane. Some of the resulting traces have SNR $<10$ and produce large errors in bulk velocity and temperature; they are discarded for the purpose of this study. For this reason, the Xe I $834.9 \mathrm{~nm}$ data set does not always extend to the exit plane. The uncertainty associated with the bulk velocity is $\pm 50 \mathrm{~m} / \mathrm{s}$. The uncertainty associated with the temperature ranges from 15-20\%, with the temperature of the wider (hotter) VDFs having lower uncertainty than that of the narrower VDFs. Only one set of representative error bars will be drawn for most plots to avoid clutter.

\section{B. Experimental Results Along Channel Centerline}

Figure $\underline{5}$ shows the most probable velocity along the thruster channel centerline obtained in the Xe I $823.4 \mathrm{~nm}$ LIF experiment for the tested operating conditions. The data set stops at the exit plane for the two $30 \mathrm{mg} / \mathrm{s}$ conditions because the SNR fell below 10 . In general, the neutrals increase in velocity as they approach the acceleration zone, which was found to typically center around the exit plane in a previous LIF study [21].

Figure 6 shows the relative density of the excited-state neutrals. Note that less data points are shown in Fig. $\underline{6}$ than in Fig. $\underline{5}$, because for traces taken at $Z<-0.3$, the collection optics view cone is partially blocked by the outer wall. While this obstruction does not affect most probable velocity measurements, it does affect peak intensity and, by extension, relative neutral density measurements. The relative density is normalized against the maximum recorded density for each operating condition. The maximum is typically

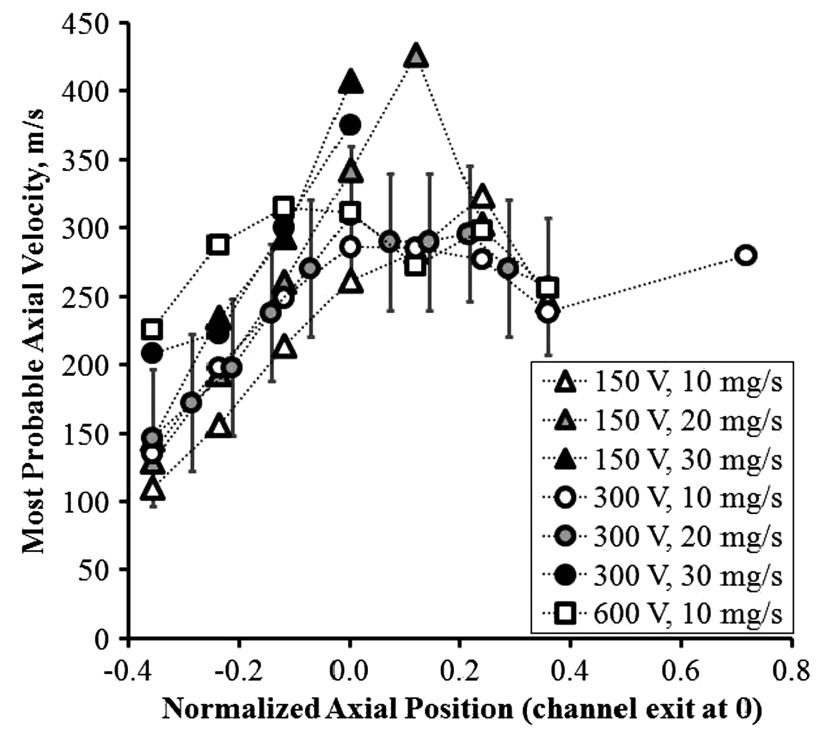

Fig. 5 Most probable velocity along the thruster channel centerline in the Xe I 823.4 $\mathrm{nm}$ experiment. 


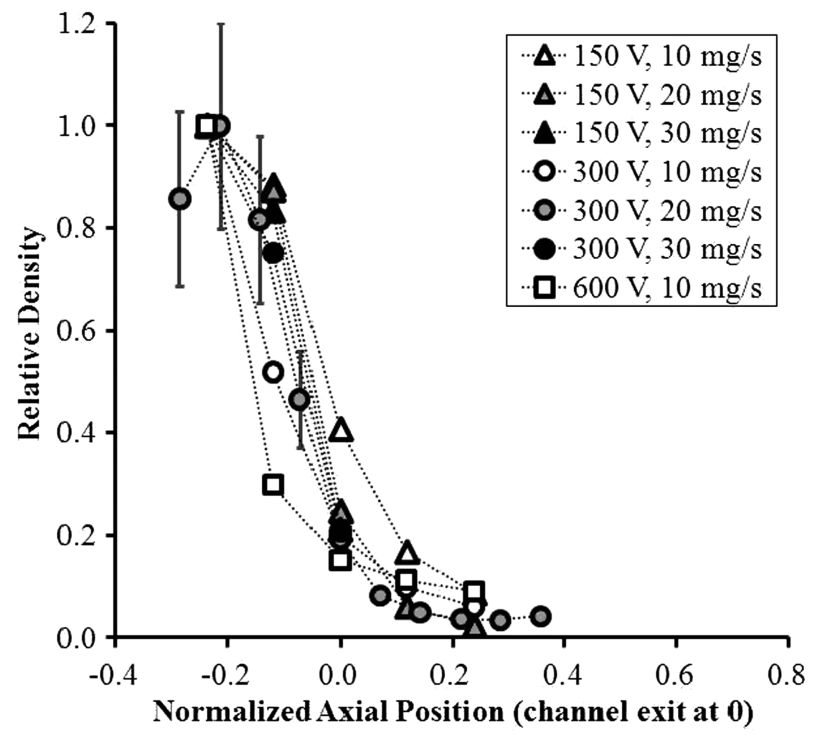

Fig. 6 Relative neutral density along the thruster channel centerline in the Xe I $823.4 \mathrm{~nm}$ experiment.

found at the furthest recorded upstream position, suggesting that the density may further increase upstream. In general, the density drops by one to two orders of magnitude over a distance that is roughly a quarter of the discharge channel length. Although the lack of data further upstream makes our deduction less conclusive, we believe we have captured the general location of the ionization zone. Previous probe studies have shown that this thruster has a mass utilization of 90-95\% across the listed operating conditions [1]. Given that the neutral velocity increases by a factor of $\sim 2$ over the interrogated distance, the overall density must drop by roughly $95-97 \%$, which matches well with the observed drop of one to two orders of magnitude in fluorescence intensity. The $10 \mathrm{mg} / \mathrm{s}$ cases may be an exception to this observation, as the drop in relative density is smaller for these cases. Electrostatic probe data confirm that the ionization zone for the 20 and $30 \mathrm{mg} / \mathrm{s}$ cases are located, at most, a third of the discharge channel length into the channel from the exit plane. The probe data also suggest that the location of the ionization zone for the $10 \mathrm{mg} / \mathrm{s}$ cases is about halfway into the discharge channel [1].

Figure 7 shows the VDFs along the channel centerline for the $300 \mathrm{~V}, 20 \overline{\mathrm{mg}} / \mathrm{s}$ condition. To make the VDFs easier to distinguish from one another, each line shape has an offset of +0.2 a.u. with respect to the preceding line shape on the legend list.

Figures 8 and 9 show the bulk axial velocity and temperature results for the Xe I $\overline{8} 34.9 \mathrm{~nm}$ LIF experiment, respectively. In general, the neutral particles start out with bulk velocities of roughly $75-125 \mathrm{~m} / \mathrm{s}$ across all conditions and gradually increase as they approach the exit plane. Combining Figs. 5 and 8, we can see that the neutrals top out at around $300 \mathrm{~m} / \mathrm{s}$ as they pass through the Hall plasma and appear to level off in velocity. There is no obviously discernible trend in the velocity with respect to the discharge voltage or the anode mass flow rate.

Surprisingly, the neutral temperature decreases as the neutrals flow downstream in the discharge channel. The neutrals start out at around 900-1600 K, which is similar to the anode temperatures we expect for this thruster. With the exception of the $300 \mathrm{~V}, 30 \mathrm{mg} / \mathrm{s}$ case, this temperature then spikes up by 100 to $600 \mathrm{~K}$, depending on operating condition, before gradually declining to $600-800 \mathrm{~K}$ near the exit plane. Because of low SNR, many of the data sets do not make it all the way to the exit plane where the bulk of the plasma is typically found. The SNR also tends to fall as one moves close to the anode, possibly because the thruster begins to obstruct part of the collection optics view cone. There appears to be a trend, where the temperature along the entire channel increases with anode mass flow rate. It does not appear that the temperature correlates strongly with the discharge voltage and the discharge power.

There is one possible explanation that fits both the temperature spike near the anode and the sensitivity of the temperature to the mass

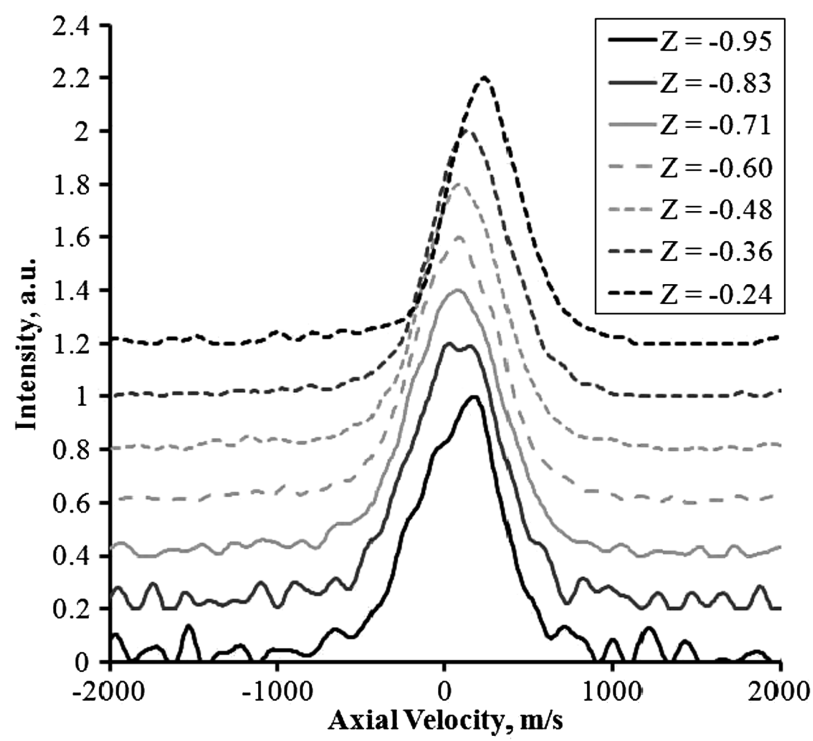

Fig. 7 Velocity distribution functions along the channel centerline for the $300 \mathrm{~V}, 20 \mathrm{mg} / \mathrm{s}$ condition.

flow rate. From basic sheath models, we know there is a sheath of about two to three times the electron temperature in front of the anode. The sheath and the accompanying presheath voltage drops are very small but noticeable in simulations like HPHall-2. Neutrals traveling through the region immediately downstream of the anode will be bombarded by ions that are energetic in comparison with the neutrals' thermal energy. The rate of energy deposition per neutral particle can be described by Eq. (1),

$$
\frac{\mathrm{d} E}{\mathrm{~d} t} \sim n_{i}\left\langle\sigma v_{i}\right\rangle \Delta E
$$

where $n_{i}$, the density of ions, should scale with the mass flow rate, $\sigma$, the collision cross section is roughly constant, $v_{i}$, the average ion velocity scales as the square root of the electron temperature, and $\Delta E$ is the average energy deposited per collision, which should scale with the electron temperature. The overall scaling can be written as Eq. (2):

$$
\frac{\mathrm{d} E}{\mathrm{~d} t} \sim \dot{m} T_{e}^{3 / 2}
$$

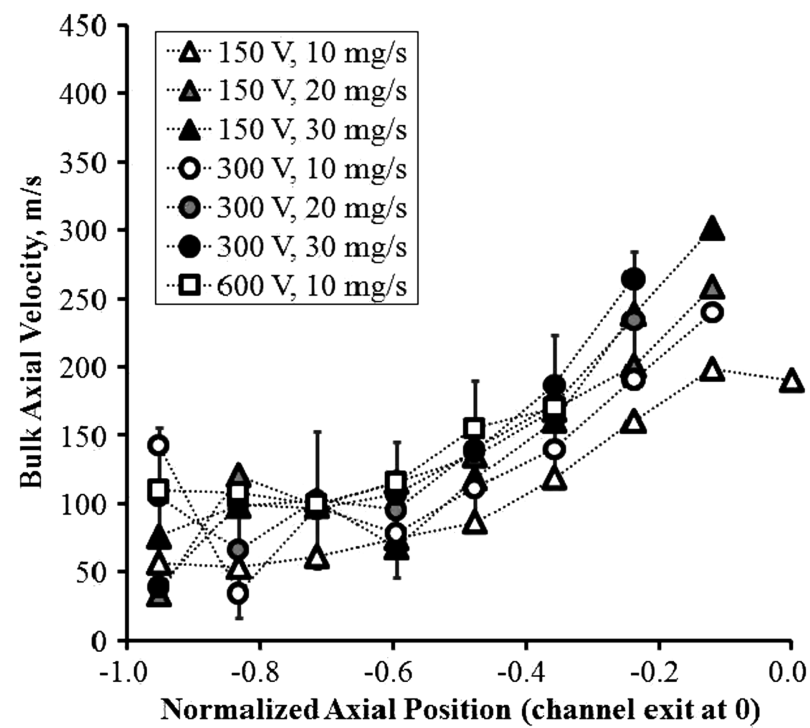

Fig. 8 Bulk axial velocity along the thruster channel centerline in the Xe I 834.9 nm experiment. 


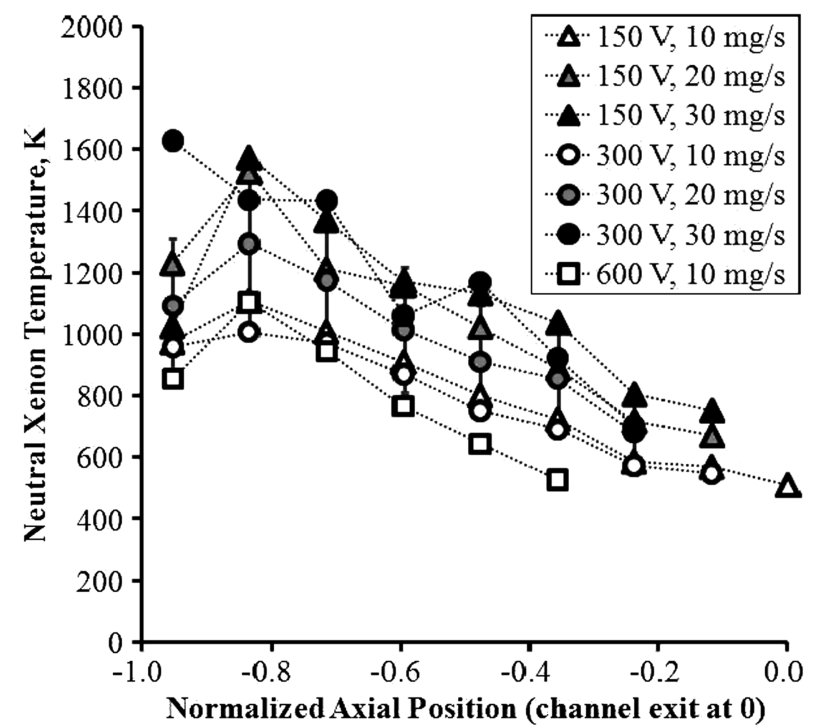

Fig. 9 Neutral xenon temperature along the channel centerline in the Xe I $834.9 \mathrm{~nm}$ experiment.

Based on the results of a fast reciprocating probe study [1], we conjecture that the electron temperature deep in the discharge channel is not sensitive to the discharge voltage. Physically, this assumption is supported by the fact that the xenon excitation and ionization cross sections are almost steplike functions with sharp cutoffs at $\sim 8$ and $\sim 12 \mathrm{eV}$, respectively. Any high-energy electron has a significant chance of exciting and ionizing neutral gas and becoming a low-energy electron. Deep in the Hall thruster channel, the electric field is relatively small, so it is possible that there are enough collisions to thermalize the electrons whenever they pick up too much energy. On the other hand, ion density scales with ion current if we assume a constant ion velocity deep within the channel, which is reasonable if the electron temperature does not change much. From these assumptions, it follows that the amount of neutral heating is more sensitive to the mass flow rate than the discharge voltage. The neutral temperature spikes from this heating, then it drops back down as the neutrals thermalize with the channel wall. Since this simple explanation involves several assumptions, we do not expect it to match the experimental results perfectly, but it does give a possible explanation for the observed trend in the neutral temperature.

Figure 10 shows a composite plot of the velocity, relative density, and temperature data for the two experiments at $300 \mathrm{~V}$ and $20 \mathrm{mg} / \mathrm{s}$.
Also shown is singly charged xenon LIF data from a previous study pointing out the location of the acceleration zone and the bulk of the plasma [21]. Density, temperature, and singly charged xenon bulk velocity are normalized against their respective maximum recorded value (left vertical axis). Neutral bulk velocity and most probable velocity measurements are plotted using the right vertical axis. From this figure, we see that the majority of the Xe I $834.9 \mathrm{~nm}$ measurements are upstream of the main ionization zone. We can also see that the peak and bulk neutral velocity measurements from the two experiments agree fairly well, given the expected uncertainty of $\pm 50 \mathrm{~m} / \mathrm{s}$.

With Fig. 10, it becomes easier to see what kind of physical explanation will give rise to the observed velocity and temperature trends. There are a number of explanations for the acceleration and cooling of neutrals well ahead of the bulk plasma. The most obvious effect is the thermalization of the neutrals with the wall. In the freemolecular limit, the neutrals only collide with the wall. Since neutral xenon has a very high accommodation factor when striking the boron nitride wall [22], they will leave at essentially the wall temperature and radiate out isotropically. In this framework, the acceleration of neutrals can be explained by the fact that, near the exit, most of the neutrals came from the wall at a relatively small angle with respect to the thrust axis so that more of their velocity is in the axial direction than particles found halfway between the anode and the channel exit. For this thruster, we expect a wall temperature of about 700-1000 K [17]. At these wall temperatures, the mean thermal speed is $330-400 \mathrm{~m} / \mathrm{s}$, which matches well with the observed terminal velocity. This mechanism of wall thermalization can also explain the observed cooling effect as the neutrals deposit thermal energy into the wall. In the continuum limit, there are still some wall effects, but the self-thermalization effect is expected to dominate. The hot but slow neutrals collide with each other, and the resulting pressure causes expansion. These collisions lead to the conversion of thermal energy into directed kinetic energy due to the geometry of the channel. This self-thermalization effect is essentially a hydrodynamic effect.

Another possibility for explaining neutral acceleration is simply that the slower neutrals spend more time in the discharge channel than the faster neutrals and are more likely to be ionized. This phenomenon, which will be called selective ionization for the remainder of this paper, can also contribute to neutral cooling. If we imagine a wide (high temperature) Maxwellian distribution and selectively remove the low-velocity population, the distribution will become more narrow (low temperature) and is thus cooled. Given that neutral acceleration and cooling are observed well ahead of the ionization zone for four out of the seven operating conditions, we conjecture that both thermalization and selective ionization are

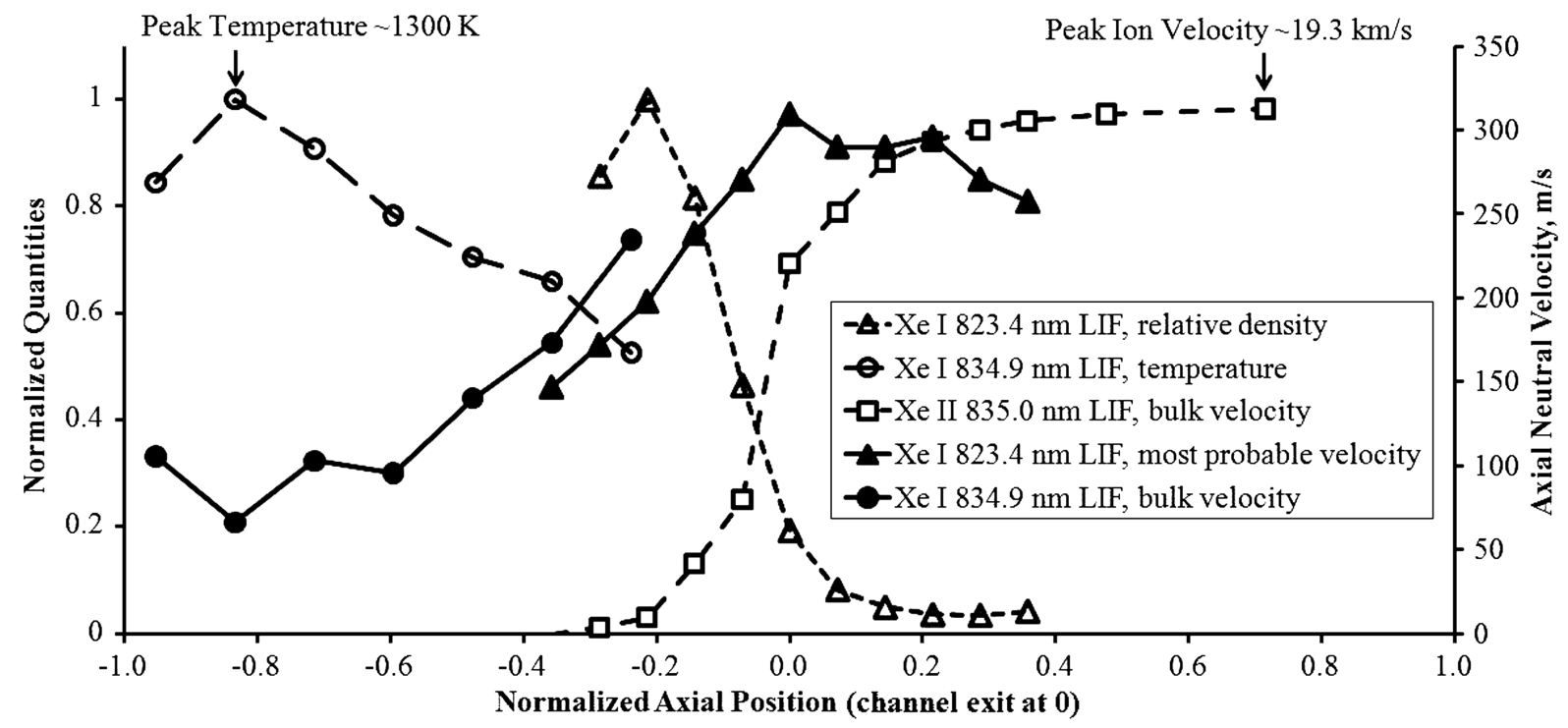

Fig. 10 Composite data plot for the $300 \mathrm{~V}, 20 \mathrm{mg} / \mathrm{s}$ operating condition. 
important for determining neutral velocity. Most likely, thermalization is more important at locations upstream of the ionization zone, and selective ionization is more important in the ionization zone. However, it is the velocity with which the neutrals enter the ionization zone that, in principle, determines residence time and affects mass utilization. In that respect, accounting for thermalization may be more important than selective ionization when simulating the neutrals.

A third explanation for neutral acceleration is charge-exchange effects. However, this effect is expected to be relatively small near the anode, where there are few fast ions, and small near the exit plane, due to the mean free path being longer than the channel length.

Since the velocimetry measurements cannot easily distinguish the effects of thermalization and ionization, we now turn to simulations to compare the behavior of neutral gas, with and without the plasma on.

\section{Comparison of Experimental and Simulation Results Along Channel Centerline}

In this section, we will show simulation results with the plasma turned on and off in an attempt to separate the effects associated with thermalization from those associated with ionization. We try to keep as many settings as possible the same between the plasma-on and plasma-off cases. In particular, by using wall and anode temperatures from plasma-on simulations in the plasma-off cases, we hope to identify the behavior of the neutral gases due only to the influence of thermalization effects in the absence of selective ionization.

Since we approximated the anode and wall temperatures when setting the boundary conditions for our simulations, it is good to see how sensitive the simulation results are to these two temperatures. Figures 11 and 12 show the results of the sensitivity study for bulk axial velocity along the channel centerline as functions of the wall and anode temperatures, respectively, in MONACO. In these figures, $T w$ is the wall temperature and $T a$ is the anode temperature. We see that the bulk axial velocity of the neutrals at the exit plane varies by about $135 \mathrm{~m} / \mathrm{s}$ for an $800 \mathrm{~K}$ change in wall temperature and varies by about $90 \mathrm{~m} / \mathrm{s}$ for an $800 \mathrm{~K}$ change in anode temperature. As expected, the anode temperature has a greater influence on the neutral velocity near the anode, while the wall temperature has a greater influence near the exit plane. For the purpose of comparing simulation and LIF results, finding the anode and wall temperatures to within $100 \mathrm{~K}$ is sufficient.

The apparent acceleration of the neutral gas can be explained if we assume the neutral velocity in the channel is primarily determined by thermalization with the geometry. To begin, let us look at the

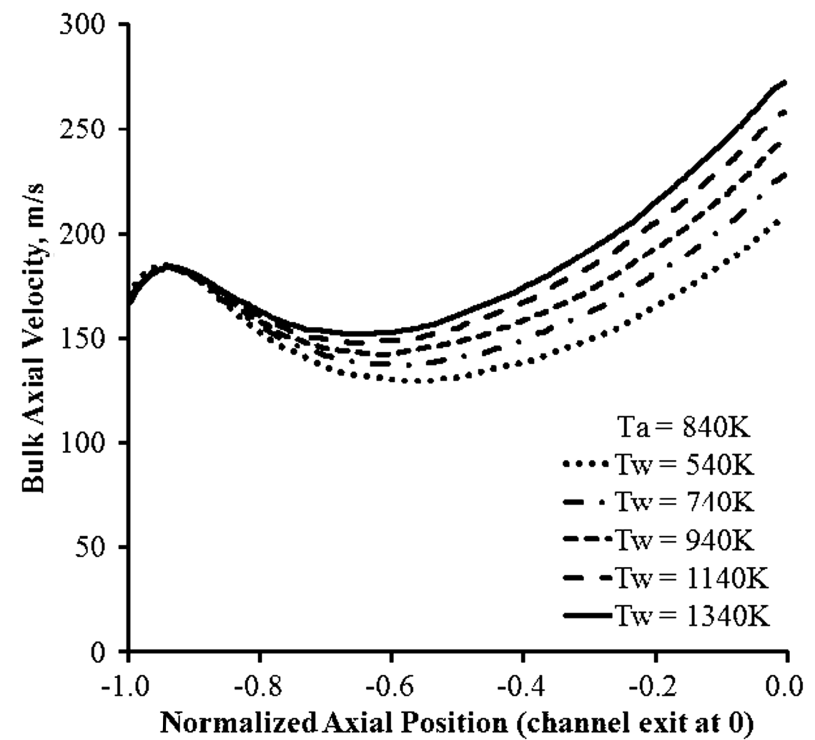

Fig. 11 MONACO sensitivity study of bulk axial velocity to wall temperature along the channel centerline.

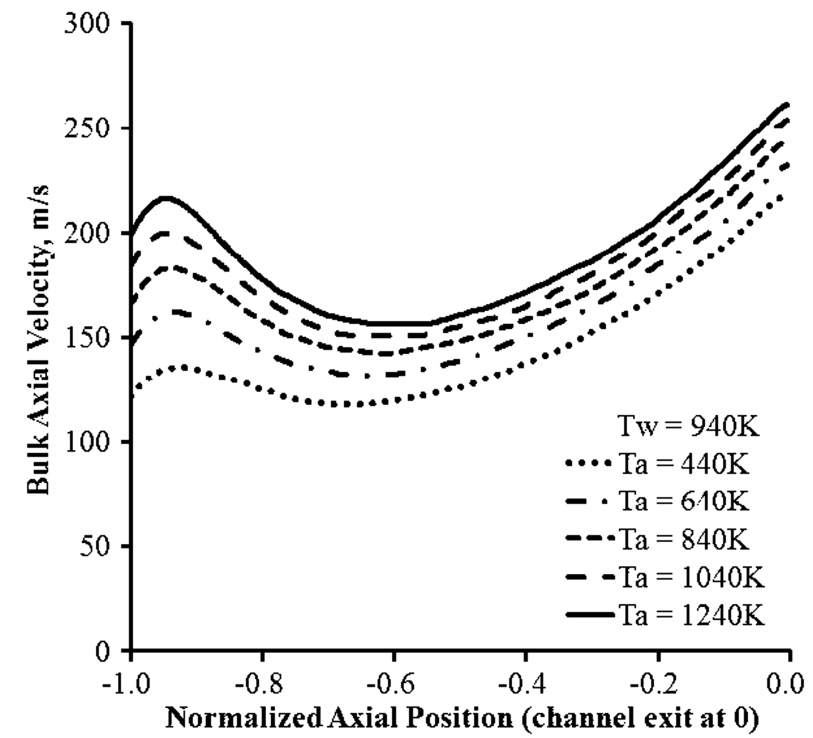

Fig. 12 MONACO sensitivity study of bulk axial velocity to anode temperature along the channel centerline.

behavior of rarefied gas in between a pair of parallel plates, as illustrated in Fig. 13. The Hall thruster channel can be approximated as a pair of parallel plate, because the channel width is much less than the channel radii. Assuming no neutral-neutral collisions and an accommodation factor of unity, the neutrals collide and thermalize only with the wall. They are then reemitted from the wall in an isotropic manner. Neutral gas velocity measured at point A will have a large negative- $z$ component because, from the perspective of that point, all neutral gas particles are coming from the positive$z$ direction. At point $\mathrm{B}$, the $z$ component of all gas particles coming from the two walls cancels, and the net measured velocity in the $z$ direction is zero. Similarly, we find that there is a large positive$z$ velocity at point $C$. Thus, it will appear as if the $z$ component of the neutral gas velocity has undergone positive- $z$ acceleration, going from the left of the diagram toward the right.

Returning to the simulation results (Figs. 11 and 12), we see a small initial bump in the axial neutral velocity just downstream of the anode that is most likely due to expansion of the flow into free space. As we move further downstream, the jet of neutral particles from the anode will diffuse out. and the fraction of neutral particles along the channel centerline that comes from the walls will increase. An initial deceleration in axial velocity in the upstream half of the channel is seen, because particles that come from the walls have a negative axial component in that half of the channel. The neutral gas then appears to accelerate due to the geometry. This phenomenon is the basis for the view factor approach, traditionally used to handle purely freemolecular flow, which has been proposed by Katz and Mikellides as a simpler way to treat neutral flow in Hall and ion thrusters [23]. Of course, the flow is actually slightly collisional, so we do not expect the simulation results to be in perfect agreement with free-molecular flow behavior.

Since we are using two different PIC codes to study this neutralflow problem, we also compared the results between the two codes

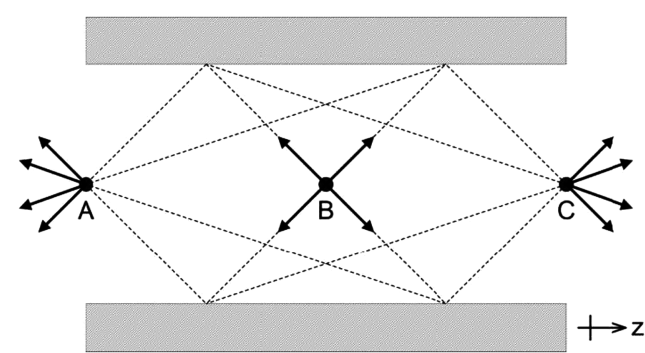

Fig. 13 Diagram of the behavior of rarefied gas in between a pair of finite parallel plates. 


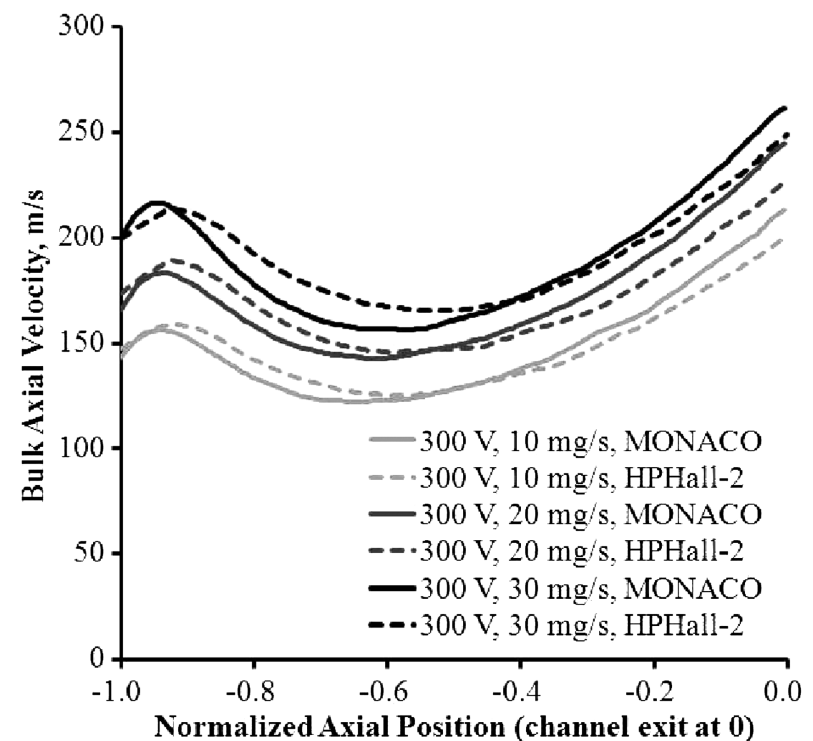

Fig. 14 Comparison of bulk axial velocity results between MONACO and HPHall-2.

for identical boundary conditions. Figure 14 shows this comparison for the $300 \mathrm{~V}$ and 10, 20, and $30 \mathrm{mg} / \mathrm{s}$ operating conditions with the hot anode and walls, but without the plasma on. The two codes agree to within $25 \mathrm{~m} / \mathrm{s}$ and predict the same general trends.

Having validated the two codes against each other, we will now compare the HPHall-2 plasma-on cases against plasma-off cases. This is shown in Fig. 15. Figure 15 shows that having the plasma on greatly increases the bulk axial velocity of the neutrals in the downstream half of the channel. The greatest increases in bulk velocity occur in locations roughly corresponding to the ionization zone and peak out near the exit plane. The drop in neutral velocity past the ionization zone may be due to the incorporation of fresh, slow neutrals coming from the wall. The trends in Fig. 15 are easiest to explain if we assume selective ionization is at work; this effect would lead to apparent neutral acceleration near the exit plane, particularly in the ionization zone. The combined simulation results from HPHall-2 and MONACO indicate that both thermalization and

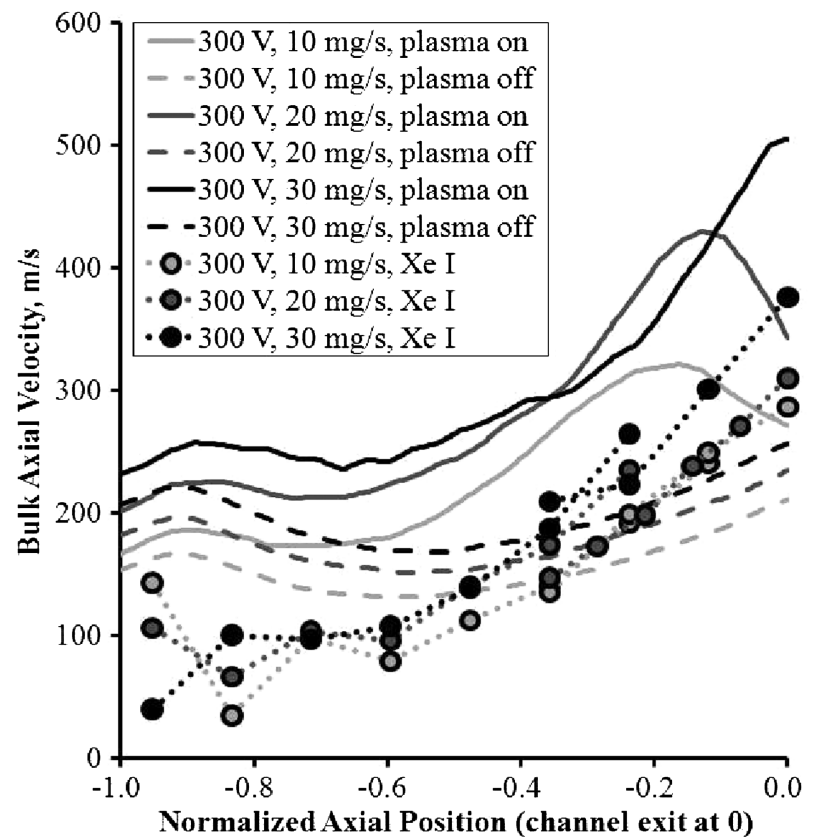

Fig. 15 Comparison of bulk axial velocity along channel centerline between the two LIF tests and the HPHall-2 simulations. selective ionization are important in determining the bulk axial velocity of the neutrals in the channel of a magnetic-layer Hall thruster.

Figure 15 also compares the LIF results to the HPHall-2 simulation results. Several trends can be seen in this plot. First, the measured bulk axial velocities show very similar trends to the HPHall-2 plasma-on simulation results, except that the measured values are roughly $100 \mathrm{~m} / \mathrm{s}$ lower and do not exhibit neutral deceleration near the channel exit. Second, the measured bulk axial velocity is closer to the plasma-off than the plasma-on simulation results, but it exhibits a noticeably higher acceleration than the plasma-off simulation results. Third, the sensitivity of the measured bulk axial velocity to operating conditions is more similar to that found in the plasma-off than the plasma-on simulation results. We can conclude from Fig. 15 that, in an actual Hall thruster, having the plasma on greatly increases the amount of neutral acceleration toward the downstream half of the channel, where the bulk plasma is found. We can also conclude that our simulations have accurately captured the amount of acceleration that the plasma induces on the neutral gas. The discrepancies between the HPHall-2 simulation and the LIF results show that, although we have made great advances in simulating Hall thruster neutral flow, there is still much that we do not fully understand. More neutral-flow velocimetry studies should be performed in order to confirm that the discrepancy is real.

We list next some possible explanations for the observed discrepancies between the measurements and the simulations. Our estimate of the channel and anode wall temperatures may be incorrect, as they were based on an empirical equation for a different $5 \mathrm{~kW}$ Hall thruster [17]. Although we assumed the anode and channel temperatures are uniform in our simulations, the test article has a temperature gradient. To see how this may be important, let us consider the neutral population just downstream of the anode. From the perspective of this region, neutral particles coming in from the section of the channel wall close to the channel exit will have a large component of their velocity in the negative axial direction. If the section of the channel wall from where these particles originate is hotter than we assumed, the axial component of these particles will be larger than we simulated in the negative axial direction, thus giving rise to a lower average velocity in the positive axial direction.

\section{Experimental and Simulation Results Across Channel Exit Plane}

Figure 16 shows the VDFs, measured by LIF, across the thruster exit plane for the $300 \mathrm{~V}, 20 \mathrm{mg} / \mathrm{s}$ condition. In order to make the velocity distribution functions easier to distinguish from one another, each line shape has an offset of +0.2 a.u. with respect to the preceding line shape on the legend list.

Figure 17 shows the collected LIF data across the thruster exit plane at the $300 \mathrm{~V}, 20 \mathrm{mg} / \mathrm{s}$ operating condition. The Xe I $834.9 \mathrm{~nm}$ data set stops at around $80 \%$ across the width of the channel due to an unplanned thruster shutdown that happened while the next data point was being taken. Although not conclusive, the velocity seems to be lower, and the temperature higher, near the walls than at the center. The fact that the velocity profile is not a parabola but is only slightly curved near the wall suggests the flow is transitional. But since there is a measurable difference in near-wall properties, there may be neutral-flow boundary layers close to the walls. The temperature is higher near the wall than at the center, most likely because near the exit, the walls receive an especially large heat load from ion impingement. In contrast, most of the neutrals in the center come from upstream locations that are colder compared with the wall near the exit. The neutral density is over three times as high near the channel wall compared with in the center of the channel. This is most likely a result of the fact that the bulk of the Hall current is confined toward the center and ionizes the neutrals that pass through the center more effectively. Note that the near-wall relative neutral density may be even higher, since we could not quite reach the walls in our density measurements. The LIF and the HPHall-2 results show the same basic profile, with the HPHall-2 results being more bowl-shaped than the LIF results. Given the measurement uncertainty, the two results match well. 


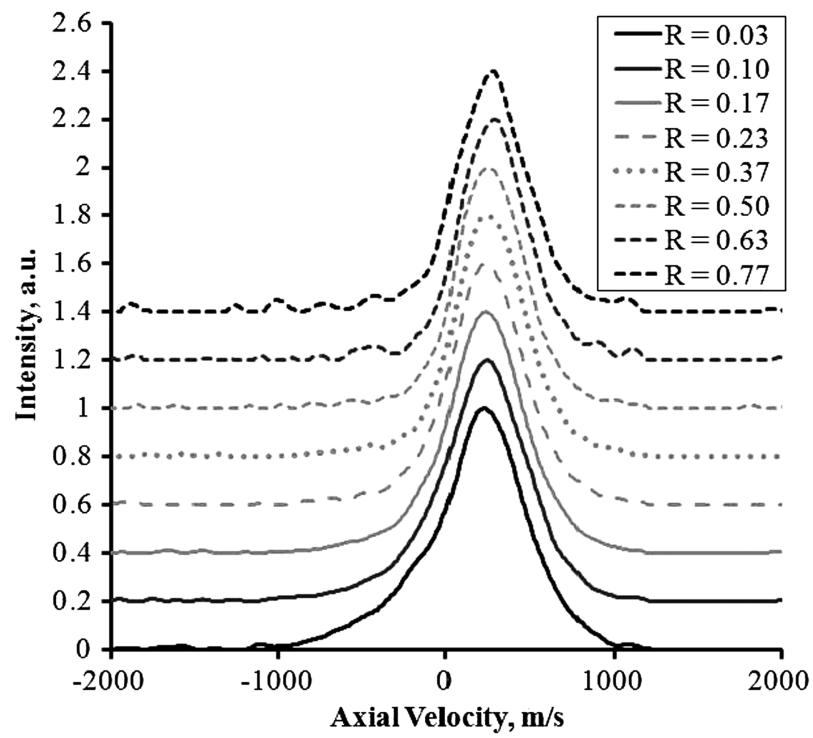

Fig. 16 Velocity distribution functions across the thruster exit plane for the $300 \mathrm{~V}, 20 \mathrm{mg} / \mathrm{s}$ condition.

\section{E. Flow Regime}

The flow regime of the neutral flow can be determined by the Knudsen number as described in Eq. (3):

$$
K n \equiv \lambda / L \quad K n \ll 1, \text { Continuum }
$$

$K n \gg 1$, Free molecular

where $\lambda$ is the mean free path of the particles and $L$ is the characteristic length scale. For neutral flow in a Hall thruster, $L$ is typically the width or the length of the discharge channel. A continuum flow in a Hall thruster can be described by the classic solution to the Poiseuille flow problem [24]. The flow at the exit plane should resemble a symmetric parabola with maximum velocity at the center. On the other hand, if the flow is free molecular, the velocity of the exiting neutrals depends only on the wall or anode temperatures from which they were reemitted after collision. In a typical magneticlayer thruster, the anode is in contact with the base of the ceramic channel. It has been shown that the temperature of the anode is typically within $100 \mathrm{~K}$ of the temperature of the channel wall, which for typical channel temperatures is a $10-15 \%$ difference [17]. Since the average velocity of thermalized particles has a square-root dependence on temperature, we can expect all the particles to exit the channel with roughly the same temperature and bulk velocity. The resulting bulk velocity profile at the exit plane should be fairly uniform.

From the results, we see that the flow regime is not continuum in the greater part of the channel, as evidenced by a lack of parabolic velocity profile at the exit plane. It also does not appear to be fully free molecular, since there is evidence of boundary-layer-like behavior. To determine more precisely which regime the flow is in, the best way is to calculate the collisional mean free path and the Knudsen number. To calculate the collisional mean free path, we need to estimate the neutral density, which can be calculated by using Eq. (4):

$$
n_{n}=\left(1-f_{i}\right) \frac{\left(\dot{m} / m_{X e}\right)}{A_{C}\langle u\rangle}
$$

where $n_{n}$ is the neutral density, $f_{i}$ is the fraction of xenon from the anode that has been ionized at the given location, $\dot{m}$ is the anode mass flow rate, $m_{X e}$ is the mass of xenon, $A_{C}$ is the channel cross sectional area, and $\langle u\rangle$ is the bulk axial velocity. To simplify our calculation, we will only use Eq. (4) in the region upstream of where the ionization zone should reside and assume $f_{i}=0$. We restrict our calculations to the data points extending from the anode to halfway between the anode and the exit plane. Since the bulk axial velocity is roughly constant in this region, we will average the LIF results for each data set to obtain the average neutral density. To calculate the Knudsen number, we let $L$ be the length of the channel and calculate the mean free path via Eq. (5):

$$
\lambda=\frac{1}{\sqrt{2} n_{n} \sigma}
$$

where $\lambda$ is the mean free path, $\sqrt{2}$ is to account for collisions between two particles of the same Maxwellian-like distribution, and $\sigma$ is the collisional cross section. The van der Waals radius for neutral xenon is $\sim 2 \AA$ [25], so the effective cross-section radius is $\sim 4 \AA$.

Table 2 summarizes the results of the calculations. As expected, the neutral density increases with anode mass flow rate. The Knudsen number ranges from 0.23 to 1.02 in the upstream half of the channel, which means the flow regime there is transitional [26]. Since we also know from our relative density measurements that the neutral density is reduced by about two orders of magnitude due to ionization in the downstream half of the channel, we can deduce that the flow regime becomes free molecular near the exit plane. Knudsen numbers calculated in the same fashion using HPHall-2 plasma-on data are also shown for comparison.

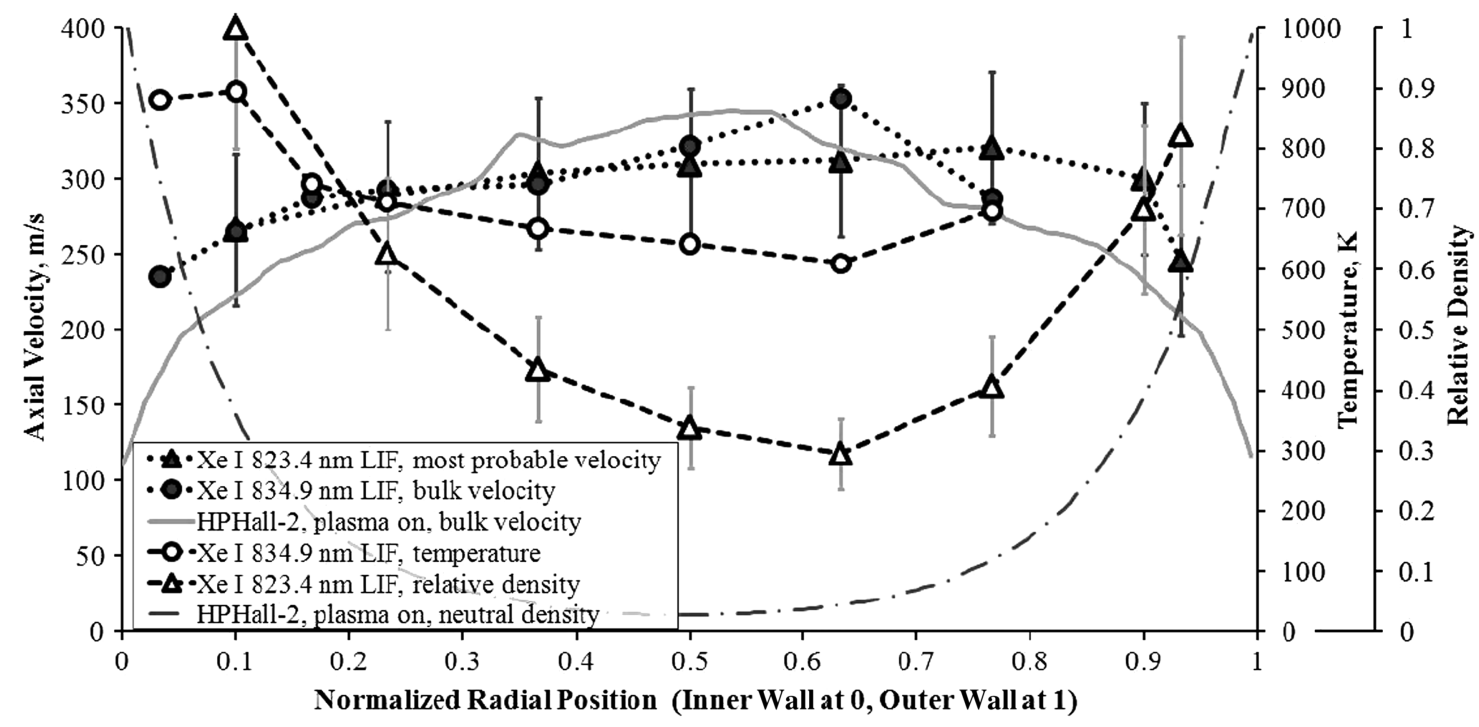

Fig. 17 Comparison of experimental data to HPHall-2 results across the thruster exit plane for the $300 \mathrm{~V}, 20 \mathrm{mg} / \mathrm{s}$ operating condition. 
Table 2 Calculated neutral-flow properties

\begin{tabular}{ccccc}
\hline \hline & & \multicolumn{3}{c}{ Average upstream value } \\
\cline { 3 - 5 } $\begin{array}{c}\text { Discharge } \\
\text { voltage, V }\end{array}$ & $\begin{array}{c}\text { Anode mass } \\
\text { flow rate, } \\
\mathrm{mg} / \mathrm{s}\end{array}$ & $\begin{array}{c}\text { Density, } \\
\mathrm{m}^{-3}\end{array}$ & $\begin{array}{c}\text { Knudsen } \\
\text { number }\end{array}$ & $\begin{array}{c}\text { HPHall-2 } \\
\text { plasma-on } \\
\text { Knudsen number }\end{array}$ \\
\hline 150 & 10 & $5.0 \mathrm{e}-19$ & 0.57 & \\
150 & 20 & $8.7 \mathrm{e}-19$ & 0.33 & \\
150 & 30 & $1.1 \mathrm{e}-20$ & 0.26 & 0.82 \\
300 & 10 & $4.5 \mathrm{e}-19$ & 0.64 & 0.51 \\
300 & 20 & $6.8 \mathrm{e}-19$ & 0.42 & 0.37 \\
300 & 30 & $1.3 \mathrm{e}-20$ & 0.23 & \\
600 & 10 & $2.8 \mathrm{e}-19$ & 1.02 & \\
\hline \hline
\end{tabular}

\section{Conclusions}

Two LIF experiments examining xenon neutrals using the Xe I 823.4 and $834.9 \mathrm{~nm}$ transitions have been performed. In general, the particle velocity starts out at around $100 \mathrm{~m} / \mathrm{s}$ at the anode and rises up to $300 \mathrm{~m} / \mathrm{s}$ at the exit plane. Equivalent Maxwellian temperatures calculated from FWHM velocity data of the extracted VDFs show a cooling effect as the neutral particles move downstream. Several physical effects, including thermalization and selective ionization, are provided as possible explanations for the trends. Radial sweeps across the exit plane reveal velocity and temperature boundary layers roughly a few millimeters thick for the $300 \mathrm{~V}, 20 \mathrm{mg} / \mathrm{s}$ operating condition. The neutral density is found to be at least a few times higher near the wall than at the center of the discharge channel for this operating condition.

Comparisons between LIF, HPHall-2, and MONACO results provide support for thermalization and selective ionization as the primary mechanisms responsible for neutral acceleration. Although the LIF data along the thruster channel centerline are consistently $\sim 100 \mathrm{~m} / \mathrm{s}$ lower than the HPHall-2 plasma-on results, they display similar trends.

Calculated Knudsen numbers indicate the flow regime is transitional in the upstream half of the channel for all operating conditions and free-molecular downstream of the ionization zone.

When the various pieces of the results found in this paper are put into a coherent picture, it is seen that the Hall thruster neutral flow is transitional and heavily influenced by heating and ionization processes. However, it is unclear to what extent each of these neutralflow mechanics needs to be modeled in simulations. Thus, further neutral-flow studies for other Hall thrusters are recommended to better understand the relative importance of various Hall thruster neutral-flow mechanics and to check for discrepancies between experimental and simulation results.

\section{Acknowledgments}

This research is funded by the U.S. Air Force Office of Scientific Research (AFOSR) grant FA9550-06-1-0105. The authors would like to acknowledge and thank the AFOSR and Mitat Birkan, the Project Manager, for this grant. The authors would also like to thank Iain D. Boyd and Jonathan Burt for the use of the MONACO code. Portions of the research described in this paper were carried out at the Jet Propulsion Laboratory, California Institute of Technology, under a contract with the NASA.

\section{References}

[1] Reid, B. M., "The Influence of Neutral Flow Rate in the Operation of Hall Thrusters," Ph.D. Dissertation, Aerospace Engineering, Univ. of Michigan, Ann Arbor, MI, 2008.

[2] Cedolin, R. J., Hargus, W. A., Hanson, R. K., and Cappelli, M. A., "Laser-Induced Fluorescence Diagnostics for Xenon Hall Thrusters," 32nd AIAA/ASME/SAE/ASEE Joint Propulsion Conference and Exhibit, Buena Vista, FL, AIAA Paper 1996-2986, July 1996.

[3] Hargus, W. A. Jr., "Laser-Induced Fluorescence of Neutral Xenon in the Near Field of a $200 \mathrm{~W}$ Hall Thruster," 41st AIAA/ASME/SAE/ASEE Joint Propulsion Conference, Tucson, AZ, AIAA Paper 2005-4400, July 2005 .
[4] Hargus, W. A. Jr., and Cappelli, M. A., "Interior and Exterior LaserInduced Fluorescence and Plasma Measurements within a Hall Thruster," Journal of Propulsion and Power, Vol. 18, No. 1, Jan.Feb. 2002, pp. 159-168. doi: $10.2514 / 2.5912$

[5] Eckbreth, A. C., Saturation Approach to Quenching, in Laser Diagnostics for Combustion Temperature and Species, Prentice-Hall, Englewood Cliffs, NJ, 1996, pp. 403-413.

[6] Candler, C., Atomic Spectra and the Vector Model, 2nd ed., Van Nostrand, Princeton, NJ, 1964, pp. 313-315.

[7] Hill, E. L., "Relative Intensities in Nuclear Spin Multiplets," Proceedings of the National Academy of Sciences of the United States of America of the United States of America, Vol. 15, No. 10, Oct. 1929, pp. 779-784. doi:10.1073/pnas.15.10.779

[8] D’Amico, G., Pesce, G., and Sasso, A., "High Resolution Spectroscopy of Stable Xenon Isotopes," Hyperfine Interactions, Vol. 127, Nos. 1-4, Aug. 2000, pp. 121-128. doi:10.1023/A:1012666811805

[9] Suzuki, M., Katoh, K., and Nishimiya, N., "Saturated Absorption Spectroscopy of Xe Using a GaAs Semiconductor Laser," Spectrochimica Acta, Part A: Molecular And Biomolecular Spectroscopy, Vol. 58, No. 11, Aug. 2002, pp. 2519-2531. doi:10.1016/S1386-1425(02)00069-0

[10] Jackson, D. A., and Coulombe, M. C., "Isotope Shifts in the Arc Spectrum of Xenon," Proceedings of the Royal Society of London A, Vol. 338, No. 1614, June 1974, pp. 277-298. doi:10.1098/rspa.1974.0086

[11] Huang, W., and Gallimore, A. D., "Laser-Induced Fluorescence Study of Neutral Xenon Flow Evolution Inside a 6-kW Hall Thruster," 31st International Electric Propulsion Conference, Ann Arbor, MI, Electric Rocket Propulsion Soc. Paper 2009-087, Fairview Park, OH, Sept. 2009.

[12] Dushman, S., Scientific Foundations of Vacuum Technique, 1st ed., Wiley, New York, 1958, Chap. 4.

[13] Smith, T. B., Huang, W., Ngom, B. B., and Gallimore, A. D., "Optogalvanic and Laser-Induced Fluorescence Spectroscopy of the Zeeman Effect in Xenon," 30th International Electric Propulsion Conference, Florence, Italy, Electric Rocket Propulsion Soc. Paper 2007-229, Fairview Park, OH, 17-20 Sept. 2007.

[14] Fife, J. M., "Hybrid-PIC Modeling and Electrostatic Probe Survey of Hall Thrusters," Ph.D. Dissertation, Aeronautics and Astronautics, Massachusetts Inst. of Technology, Boston, 1999.

[15] Parra, F. I., Ahedo, E., Fife, J. M., and Martinez-Sanchez, M., "A TwoDimensional Hybrid Model of the Hall Thruster Discharge," Journal of Applied Physics, Vol. 100, No. 2, July 2006, Paper 023304. doi:10.1063/1.2219165

[16] Hofer, R. R., Katz, I., Mikellides, I. G., Goebel, D. M., Jameson, K. K., Sullivan, R. M., and Johnson, L. K., "Efficacy of Electron Mobility Models in Hybrid-PIC Hall Thruster Simulations," 44th AIAA/ASME/ SAE/ASEE Joint Propulsion Conference and Exhibit, Hartford, CT, AIAA Paper 2008-4924, 21-23 July 2008.

[17] Mazouffre, S., Echegut, P., and Dudeck, M., "A Calibrated Infrared Imaging Study on the Steady State Thermal Behavior of Hall Effect Thrusters," Plasma Sources Science and Technology, Vol. 16, No. 1, Feb. 2007, pp. 13-22. doi:10.1088/0963-0252/16/1/003

[18] Hofer, R. R., Katz, I., Mikellides, I. G., and Castano, M. G., "Heavy Particle Velocity and Electron Mobility Modeling in Hybrid-PIC Hall Thruster Simulations," 42nd AIAA/ASME/SAE/ASEE Joint Propulsion Conference and Exhibit, Sacramento, CA, AIAA Paper 20064658, 9-12 July 2006.

[19] Dietrich, S., and Boyd, I. D., "Scalar and Parallel Optimized Implementation of the Direct Simulation Monte Carlo Method," Journal of Computational Physics, Vol. 126, No. 2, 1996, pp. 328-342. doi:10.1006/jcph.1996.0141

[20] Smith, T. B., "Deconvolution of Ion Velocity Distributions from LaserInduced Fluorescence Spectra of Xenon Electrostatic Thruster Plumes," $\mathrm{Ph} . \mathrm{D}$. Dissertation, Aerospace Engineering, Univ. of Michigan, Ann Arbor, MI, 2003.

[21] Huang, W., Drenkow, B., and Gallimore, A. D., "Laser-Induced Fluorescence of Singly-Charged Xenon Inside a 6-kW Hall Thruster," 45th AIAA/ASME/SAE/ASEE Joint Propulsion Conference and Exhibit, Denver, CO, AIAA Paper 2009-5355, 2-5 Aug. 2009.

[22] Agrawal, A., and Prabhu, S. V., "Survey on Measurement of Tangential Momentum Accommodation Coefficient," Journal of Vacuum Science and Technology A (Vacuum, Surfaces, and Films), Vol. 26, No. 4, July 2008, pp. 634-645. doi: $10.1116 / 1.2943641$ 
[23] Katz, I., and Mikellides, I. G., "A New Algorithm for the Neutral Gas in the Free-Molecule Regimes of Hall and Ion Thrusters," 31st International Electric Propulsion Conference, Ann Arbor, MI, Electric Rocket Propulsion Soc. Paper 2009-095, Fairview Park, OH, 20 24 Sept. 2009.

[24] White, F. M., Combined Couette-Poiseuille Flow Between Plates, in Viscous Fluid Flow, 3rd ed., McGraw-Hill, New York, 2006, pp. 110 112.

[25] Bondi, A., "Van Der Waals Volumes and Radii," Journal of Physical
Chemistry, Vol. 68, No. 3, March 1964, pp. 441-451. doi: $10.1021 / \mathrm{j} 100785 \mathrm{a} 001$

[26] Vincenti, W. G., and Kruger, C. H. Jr., Introductory Kinetic Theory, in Introduction to Physical Gas Dynamics, Krieger, Malabar, FL, 1965, Chap. 1.
J. Blandino Associate Editor 